\title{
The double cone: a mechanical paradox or a geometrical constraint?
}

\author{
Aurelio Agliolo Gallitto and Emilio Fiordilino \\ Dipartimento di Fisica, Università di Palermo, via Archirafi 36, I-90123 Palermo, Italy \\ E-mail: aurelio.agliologallitto@unipa.it
}

\begin{abstract}
In the framework of the Italian National Plan 'Lauree Scientifiche' (PLS) in collaboration with secondary schools, we have investigated the mechanical paradox of the double cone. We have calculated the geometric condition for obtaining an upward movement. Based on this result, we have built a mechanical model with a double cone made of aluminum and a couple of wooden rails.
\end{abstract}

In the eighteenth and nineteenth centuries, in order to illustrate Newton's laws of mechanics many apparent paradoxes were found, and therefore a variety of instruments constructed to illustrate them. These devices make it possible to perform astonishing experiments that contradict common sense. However, after deeper observation and analysis, the experiments overcome the initial sense of amazement demonstrating that the phenomena are perfectly consistent with the laws of physics.

The most popular mechanical paradox consists of a double cone, formed by joining two identical cones at their bases. The double cone is located on a pair of tilted and diverging rails. When the double cone is placed at the lower end of the rails, it spontaneously begins to move upward giving the impression of defying the universal law of gravitation. Because of this phenomenon, which seems to contradict common sense, the apparatus is often described as a mechanical paradox.

In this article, we calculate the geometric condition for obtaining an upward movement of the double cone. Based on this calculation, we have built a mechanical model with a double cone made of solid aluminum and a couple of wooden rails. This was carried out in the framework of the Italian National Plan 'Lauree Scientifiche' $(\mathrm{PLS})^{1}$ in collaboration with several secondary schools $[1,2]$.

The apparent paradox is naturally explained in the framework of the universal law of gravitation. A body in the presence of the gravitational force moves towards a minimum of the gravitational potential energy. Since the rails diverge, the centre of mass (CM) of the double cone, placed on the axis of rotation at the maximum diameter, does not rise when the entire body seems to move up; on the contrary, the CM moves downwards. During the motion of the double cone, the points of contact with the rails move towards the apex of each cone. As a result, the distance of the centre of gravity from the horizontal plane decreases. The phenomenon is thus totally consistent with Newton's laws of mechanics.

In order to understand whether the double cone moves upwards, one must calculate the variation of the height of the double-cone axis with respect to the horizontal plane after an

${ }^{1}$ For more information see www.progettolaureescientifiche. eu. 
The double cone: a mechanical paradox or a geometrical constraint?

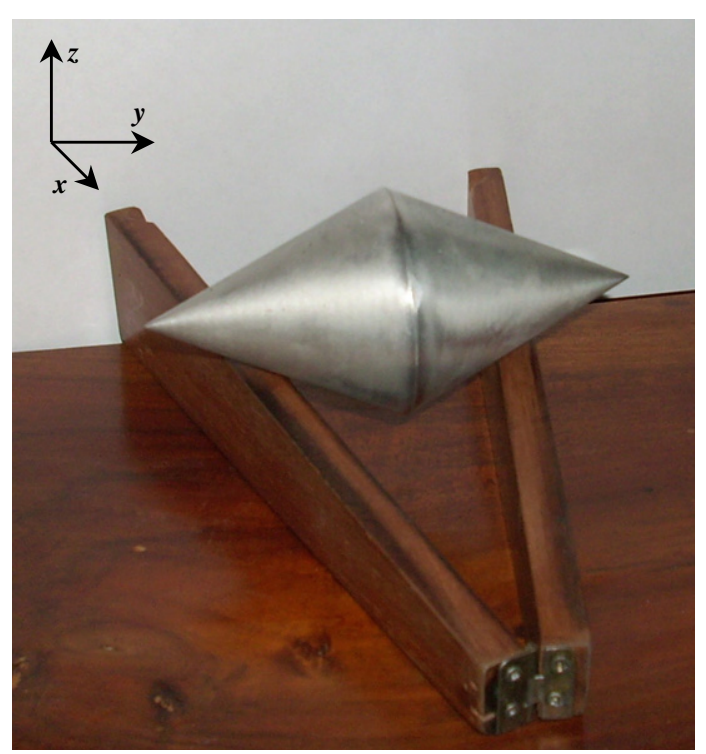

Figure 1. A picture of the double cone, along with the coordinate system.

infinitesimal horizontal displacement. Let us consider a Cartesian coordinate system with $\hat{x}$ in the horizontal direction of motion, $\hat{y}$ in the horizontal plane (orthogonal to $\hat{x}$ ) and $\hat{z}$ in the vertical direction; figure 1 shows a picture of the double cone, on which is indicated the coordinate system.

A schematic perspective of the double cone and the rails is shown in figure 2; let us call $\theta$ the semi-angle of the cone, as indicated in figure 2(a); $\psi$ the semi-angle of the rails and $\varphi$ the tilt angle of the rails, as indicated in figures 2(b) and (c), respectively.

The distance $y(x)$ between the contacts of the double cone and the rails (indicated in figure 2(a) by $\mathrm{A}$ and $\mathrm{B}$ ) increases with the displacement $\mathrm{d} x$ in the direction of motion of the double-cone axis

$$
\mathrm{d} y=y(x+\mathrm{d} x)-y(x)=\tan (\psi) \mathrm{d} x ;
$$

consequently, the level difference between the double-cone axis and the contact points decreases by the following amount

$$
\begin{aligned}
& \mathrm{d} \zeta=\zeta(y+\mathrm{d} y)-\zeta(y)=\tan (\theta) \mathrm{d} y \\
& \quad=\tan (\theta) \tan (\psi) \mathrm{d} x .
\end{aligned}
$$

On the other hand, the contact points on the rails, which are tilted by an angle $\varphi$ with respect to the horizontal plane, rise with displacement $\mathrm{d} x$ of the following amount:

$$
\mathrm{d} z=z(x+\mathrm{d} x)-z(x)=\tan (\varphi) \mathrm{d} x .
$$

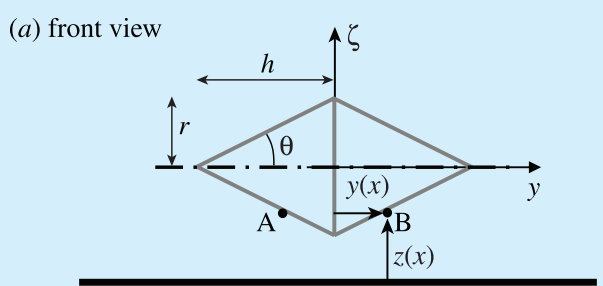

(b) top view

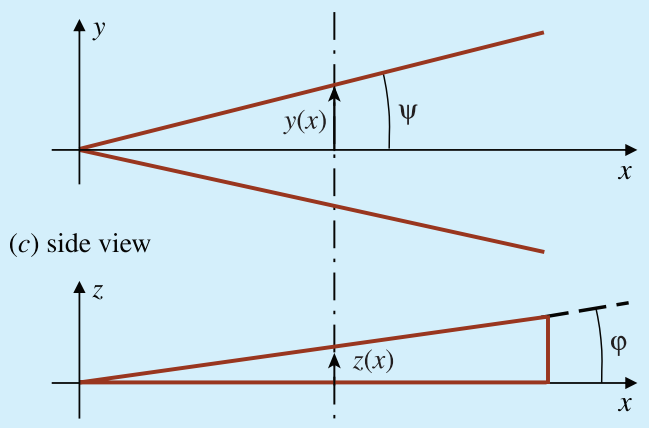

Figure 2. Schematic perspectives of the double cone and the rails: $(a)$ front view of the double cone, $(b)$ top view of the rails and $(c)$ side view of the rails.

The net vertical displacement of the double-cone axis is obtained subtracting equations (3) and (2). In order to observe an upward movement of the double cone, the following condition must be fulfilled:

$$
\mathrm{d} z-\mathrm{d} \zeta<0 \Rightarrow \tan (\varphi)<\tan (\theta) \tan (\psi) .
$$

When $\mathrm{d} \zeta>\mathrm{d} z$, the CM of the double cone moves downwards and the gravitational potential energy decreases, even if the contact points between the double cone and the rails move upwards. This gives rise to the apparent paradox that the double cone moves up on the rails.

In order to verify the condition described by equation (4), we have built a double cone made of solid aluminum and a couple of wooden rails. The dimensions of the double cone are $r=30 \mathrm{~mm}$ and $h=65 \mathrm{~mm}$, which forms an angle $\theta=$ $\arctan (r / h)=24.8^{\circ}$. The rails are tilted by an angle $\varphi=6.5^{\circ}$ and form a horizontal semi-angle $\psi=15.3^{\circ}$. The mechanical model is shown in figure 1 . Our homemade double cone works very well and, of course, satisfies the condition given by equation (4)

$$
\begin{aligned}
& \tan \left(6.5^{\circ}\right)<\tan \left(24.8^{\circ}\right) \times \tan \left(15.3^{\circ}\right) \\
& \Rightarrow 0.114<0.126 .
\end{aligned}
$$


We would remark that, on reducing the angle $\psi$ of the rails, one gets the geometric condition for the indifferent static equilibrium of the double cone on the rails, which is expected to be $\psi \approx$ $13.8^{\circ}$. We have found experimentally that the equilibrium is attained as $\psi \approx 13.3^{\circ} \pm 0.7^{\circ}$; the experimental uncertainty is due to the friction forces that contribute to keep the double cone in a steady position.

Once we have found the vertical displacement of the double-cone axis, it is possible to calculate the gravitational potential energy variation and thus the final velocity of the double cone. In order to simplify calculations, we shall assume that the condition of pure rolling is always achieved; this will prevent work by the frictional forces at the point of contact of the rails and cone. By using the dimensions of our apparatus, we calculate a maximum vertical displacement of the $\mathrm{CM} \Delta z \approx$ $2 \mathrm{~mm}$. The decrease of gravitational potential energy, $\Delta U$, will convert into kinetic energy, $\Delta K$, of the double cone. During the motion, the kinetic energy is rotational and translational; at the end, the kinetic energy will be only rotational because when the contacts approach the apexes the radius $r_{\mathrm{c}}$ reduces to zero. Then, by the assumption of pure rolling, the translational velocity $v=\omega r_{\mathrm{c}} \rightarrow$ 0 , where $\omega$ is the angular velocity. This means that the kinetic energy of the double cone will be mainly rotational. From the above consideration, one can write

$$
\Delta U=-m g \Delta z
$$

where $m$ is the mass of the double cone and $g=$ $9.81 \mathrm{~m} \mathrm{~s}^{-2}$ is the gravitational acceleration; then, from the principle of energy conservation

$$
\Delta U+\Delta K=0 \Rightarrow m g \Delta z=\frac{1}{2} I_{c m} \omega^{2},
$$

where $I_{c m}$ is the moment of inertia of the double cone given by $I_{c m}=\frac{3}{10} m r^{2}$. Simplifying the mass, one obtains

$\omega=\sqrt{\frac{20 g \Delta z}{3 r^{2}}} \approx 12 \operatorname{rad~s}^{-1} \approx 2$ revolutions s${ }^{-1}$.

It is worth noting that the value of the mass does not enter the calculation; however, its distribution in the device is very relevant since it affects the value of $I_{c m}$.

The experiment with the double cone is of value and importance from a pedagogical point of view. The students at the beginning of the study of mechanics are attracted by the paradox and, invited by their teachers, explain the phenomenon by using their knowledge of Newton's laws. By observation of the movements of the double-cone axis, they can find the real explanation. An exact calculation of the vertical displacements can be also done on the basis of the mathematical background of students.

In conclusion, a simple calculation of the displacement of the $\mathrm{CM}$ of the double cone has been done, showing that the paradox is only apparent. The CM of the double cone moves downwards while it is rolling up on the rails, thus respecting Newton's laws of mechanics. Following the geometry condition, we have successfully built a mechanical model that works very well.

\section{Acknowledgments}

The authors thank G Napoli for technical assistance. This work is done in the framework of the Italian National Plan 'Lauree Scientifiche' under the financial support of the Italian Ministry of Education, University and Research.

Received 1 April 2011, in final form 17 May 2011 doi:10.1088/0031-9120/46/6/002

\section{References}

[1] Agliolo Gallitto A 2009 Quattro Anni di Attività del Progetto Lauree Scientifiche-Fisica nell'Ateneo di Palermo Università e Scuola XIV $228-33$

[2] Fiordilino E and Agliolo Gallitto A $2010 \mathrm{Il}$ laboratorio di fisica nel Progetto Lauree Scientifiche (Rome: Aracne)

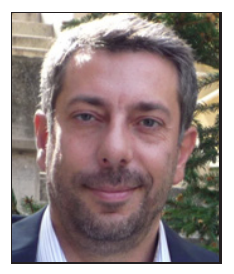

Aurelio Agliolo Gallitto is an associate professor of experimental physics at the University of Palermo, Italy. He works in the field of superconductivity at microwave frequencies. From 2008 to 2010, he was local coordinator of the National Project 'Lauree Scientifiche'.

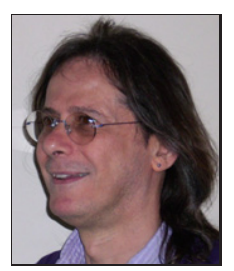

Emilio Fiordilino teaches field theory and statistical mechanics at the University of Palermo, Italy. His research is mainly on the physics of the interaction of strong electromagnetic fields with molecules and nanoparticles, but also on volcanism. He spends part of his time lecturing on quantum mechanics at high schools. 